\title{
Extremal holomorphic curves for defect relations
}

\author{
Alexandre Eremenko*
}

\begin{abstract}
Drasin's theorem describing meromorphic functions of finite order with maximal sum of deficiencies is extended to holomorphic curves in projective space. A conjecture about holomorphic curves extremal for Cartan's defect relation is discussed.
\end{abstract}

1. Introduction. We study meromorphic functions and their generalization, entire holomorphic curves in projective space, which are extremal from the point of view of value distribution theory.

The prototype of all results considered here is the following simple theorem: let $f$ be an entire function of finite order omitting 0. Then $f=\exp P$, where $P$ is a polynomial. Thus two assumptions: $f$ is extremal for Picard's theorem and satisfies a growth restriction imply very strong conclusions about behavior of $f$ near infinity: the asymptotic behavior of $\log |f|$ is very regular and the order can assume only a discrete set of values.

Before going further we recall necessary definitions from value distribution theory [13].

We consider holomorphic curves $f: \mathbf{C} \rightarrow \mathbf{P}^{n}$ where $\mathbf{C}$ is the complex line and $\mathbf{P}^{n}$ is complex projective space of dimension $n$. Denote by $\pi$ : $\mathbf{C}^{n+1} \backslash\{0\} \rightarrow \mathbf{P}^{n}$ the standard projection. Let $f=\left(f_{0}: \ldots: f_{n}\right)$ be a reduced homogeneous representation of $f$. This means that $\tilde{f}=\left(f_{0}, \ldots, f_{n}\right): \mathbf{C} \rightarrow$

*Supported by NSF grant DMS-950036. This paper was written in Norwegian Technology and Science University (NTNU, Trondheim), which the author thanks for hospitality. 
$\mathbf{C}^{n+1} \backslash\{0\}$ is a holomorphic mapping such that $f=\pi \circ \tilde{f}$. The NevanlinnaCartan characteristic is defined by

$$
T(r)=T(r, f)=\frac{1}{2 \pi} \int_{0}^{2 \pi} \log \left\|\tilde{f}\left(r e^{i \theta}\right)\right\| d \theta-\log \|f(0)\|,
$$

where $\|Z\|=\sqrt{\left|z_{0}\right|^{2}+\ldots+\left|z_{n}\right|^{2}}$ is the standard norm in $\mathbf{C}^{n+1}$. If $n=$ 1 this definition gives the classical Nevanlinna characteristic (in the form of Ahlfors). Slightly abusing the notation we will identify a meromorphic function $g=f_{1} / f_{0}$ with the one-dimensional curve $f=\left(f_{0}: f_{1}\right)$.

In this paper we consider only curves of finite lower order

$$
\rho=\liminf _{r \rightarrow \infty} \frac{\log T(r, f)}{\log r}<\infty .
$$

A hypersurface $a \subset \mathbf{P}^{n}$ is defined by a homogeneous form $P_{a}$ of some degree $d$ in $n+1$ variables. Then zeros of the entire function

$$
g_{a}=P_{a} \circ \tilde{f}
$$

are preimages $f^{-1}(a)$. Let

$$
n(r, a)=n_{f}(r, a)=\#\left\{z \in \mathbf{C}: g_{a}(z)=0,|z| \leq r\right\}
$$

be the counting function (with multiplicity) of these preimages. Then

$$
N(r, a)=\int_{0}^{r}(n(t, a)-n(0, a)) \frac{d t}{t}+n(0, a) \log r
$$

is called the averaged counting function of preimages.

The defect of $f$ at $a$ is defined by

$$
\delta(a, f)=\liminf _{r \rightarrow \infty}\left(1-\frac{N(r, a)}{d T(r, f)}\right),
$$

where $d=\operatorname{deg} P_{a}$. Defect does not change if we raise $P_{a}$ to some power.

A system of hypersurfaces is called admissible if the intersection of any $n+1$ hypersurfaces of this system is empty.

In this paper we study the extremal curves for the following defect relation proved in [9]. 
Theorem A Let $A$ be an admissible system of hypersurfaces and $f$ be a holomorphic curve, $f(\mathbf{C}) \notin \cup\{a \in A\}$. Then

$$
\sum_{a \in A} \delta(a, f) \leq 2 n
$$

If $n=1$ Theorem A coincides with the defect relation of Nevanlinna:

$$
\sum_{a \in \overline{\mathbf{C}}} \delta(a, f) \leq 2 .
$$

(Hypersurfaces in $\mathbf{P}^{1}$ are just points in $\overline{\mathbf{C}}$ and any set of points is admissible). The case of equality in (4) is described by the following theorem of D. Drasin.

Theorem B Let $f$ be a meromorphic function of finite lower order $\rho$ satisfying

$$
\sum_{a \in \overline{\mathbf{C}}} \delta(a, f)=2 .
$$

Then

(i) $2 \rho$ is an integer $\geq 2$; if in addition $\delta(a, f)=1$ for some $a \in \overline{\mathbf{C}}$ then $\rho$ is a positive integer;

(ii) $T(r, f)=r^{\rho} l(r)$, where $l$ is a slowly varying function ${ }^{1}$;

(iii) $\delta(a, f)=p(a) / \rho$, where $p: \overline{\mathbf{C}} \rightarrow \mathbf{Z}_{+}$is a integer valued function, $\sum_{a \in \overline{\mathbf{C}}} p(a)=2 \rho$.

This formulation is taken from [6] (see also [7]). In Drasin's original paper [4] a stronger assumption is made, namely that the upper limit in (1) is finite and the conclusion (ii) is not stated explicitly. Notice that (ii) in Theorem $\mathrm{B}$ is a stronger regularity condition then the existence of limit in (1).

We extend Drasin's theorem to the case of equality in (3) in arbitrary dimension.

Theorem 1 Let $f$ be a holomorphic curve in $\mathbf{P}^{n}$ of finite lower order $\rho$ and $A$ be an admissible system of hypersurfaces. If

$$
\sum_{a \in A} \delta(a, f)=2 n
$$

\footnotetext{
${ }^{1}$ in the sense of Karamata. That is $l(c r) / l(r) \rightarrow 1$ as $r \rightarrow \infty$, uniformly with respect to $c \in[1,2]$.
} 
then:

(i) $2 \rho$ is an integer $\geq 2$; if in addition $\delta(a, f)=1$ for at least one hypersurface a then $\rho$ is a positive integer;

(ii) $T(r, f)=r^{\rho} l(r)$, where $l$ is a slowly varying function;

(iii) $\delta(a, f)=p(a) / \rho$, where $p$ is a function $A \rightarrow \mathbf{Z}_{+}$and $\sum_{a \in A} p(a)=2 n \rho$;

The inequality (3) is best possible: there are admissible systems of hypersurfaces of any degree and holomorphic curves for which (6) holds. However in all such known examples the curve $f$ as well as the configuration of hypersurfaces $A$ are highly degenerate; the image $f(\mathbf{C})$ is contained in an algebraic curve $\Gamma$, and hypersurfaces from $A$ are either linear or tangent to $\Gamma$ at the intersection points. On the other hand it has never been proved that (6) implies any kind of degeneracy of $f$ or $A$, except in the case of linear hypersurfaces (hyperplanes) when (6) indeed implies that the image of $f$ is contained in a complex line [15].

Our proof of Theorem 1 gives the following result about degeneracy.

Recall that hypersurfaces $a_{1}, \ldots, a_{k}$ have normal intersection at a point $x \in \cap_{i=1}^{k} a_{i}$ if all these hypersurfaces are non-singular at $x$ and their normal vectors at $x$ are linearly independent.

Theorem 2 Assume that homogeneous polynomials defining hypersurfaces of an admissible system $A$ are irreducible and all have degrees at least 2. If (6) holds for a holomorphic curve $f$ of finite order then there are $2 n$ hypersurfaces $a_{1}, \ldots, a_{2 n} \in A$ such that at some point $Z_{0} \in \cap_{i=1}^{n} a_{i}$ as well as at some point $Z_{1} \in \cap_{i=n+1}^{2 n} a_{i}$ the intersections are not normal.

This supports the conjecture that for non-singular hypersurfaces with normal intersections of degree greater than one the defect relation (3) can be improved.

If the curve $f$ is linearly non-degenerate (that is $f(\mathbf{C})$ is not contained in any hyperplane), and hypersurfaces in an admissible system $A$ are hyperplanes, we have a stronger defect relation which is due to H. Cartan [3] (see also [13]):

$$
\sum_{a \in A} \delta(a, f) \leq n+1
$$

When $n=1$ this also coincides with (4). The study of extremal curves for (7) turns out to be much harder than the study of those extremal for (3). 
If $f$ is a linearly non-degenerate holomorphic curve of finite order and for some admissible system $A$ of hyperplanes we have

$$
\sum_{a \in A} \delta(a, f)=n+1,
$$

then the Second Main theorem of H. Cartan $[3,13]$ implies that the averaged counting function $N_{1}(f)$ of zeros of the Wronskian determinant $W\left(f_{0}, \ldots, f_{n}\right)$ has the property

$$
N_{1}(r, f)=o(T(r, f)) .
$$

Thus to make reasonable conjectures about extremal curves one has to look first at the curves satisfying

$$
N_{1}(r, f) \equiv 0 .
$$

V. P. Petrenko [16] showed that linearly non-degenerate holomorphic curves $f=\left(f_{0}: \ldots: f_{n}\right)$ of finite lower order having the property (10) are exactly those whose components $f_{j}$ can be chosen to form a fundamental system of solutions of a linear differential equation

$$
w^{n+1}+c_{n} w^{n}+\ldots+c_{0} w=0
$$

with polynomial coefficients $c_{j}$. (Case $n=1$ is due to F. Nevanlinna).

Solutions of such differential equations are known to have very regular behavior. In particular one can deduce that the lower order of $f$ coincides with its order, this number is positive and rational, and its denominator equals to the order of some permutation of a set of $n+1$ elements. One can conjecture that the same property remains true if one replaces (10) by the weaker condition (9). Such a strong result is known only in dimension 1 (see $[7])$ :

Theorem C. Let $f$ be a meromorphic function of finite lower order with the property $N_{1}(r, f)=o(T(r, f))$. Then all conclusions $(i)-($ iii $)$ of Theorem $B$ hold. In particular (9) is equivalent to (4).

In higher dimensions very little is known about extremal curves in Cartan's defect relation. The following theorem was recently proved by N. Toda [17] who improved pervious results of Noguchi and Mori. 
Theorem $\mathbf{D}$ Let $f$ be a linearly non-degenerate holomorphic curve of finite order $\rho$ in $\mathbf{P}^{n}$ and $A=\left\{a_{j}\right\}$ be an admissible system of hyperplanes. If

$$
\delta\left(a_{j}, f\right)=1, \quad 1 \leq j \leq n,
$$

and (8) holds then the limit in (1) exists and $\rho$ is a positive integer.

The assumption (12) is certainly too strong. That the situation in higher dimensions is really more complicated than in dimension 1 shows the following example.

Example. There is a holomorphic curve $f$ of order $3 / 2$ in $\mathbf{P}^{2}$ with property (9) and such that there is no admissible system of hyperplanes satisfying (8).

This example will be constructed in Section 5. Another simple example shows that when (8) is satisfied the deficiencies can be irrational. Indeed, take $f=\left(e^{z}: e^{\alpha z}: 1\right)$ with $0<\alpha<1$ and the following admissible system of hyperplanes (described by coefficients of corresponding linear forms):

$$
a_{1}=(0,1,1), \quad a_{2}=(1,1,0), \quad a_{3}=(1,0,0), \quad a_{4}=(0,0,1) .
$$

Then a direct computation shows that $\delta\left(a_{1}, f\right)=\alpha, \delta\left(a_{2}, f\right)=1-\alpha$ and $\delta\left(a_{3}, f\right)=\delta\left(a_{4}, f\right)=1$.

\section{Preliminaries}

2.1 Choice of a homogeneous representation. Sometimes we have to consider homogeneous representations $f=\left(f_{0}: \ldots: f_{n}\right)$ which are not reduced, that is $f_{j}$ are allowed to have zeros common to all of them. We always assume however that $f_{0}(0) \neq 0$. In the case of arbitrary homogeneous representation $\tilde{f}=\left(f_{0}, \ldots, f_{n}\right)$ we have

$$
T(r, f)=\frac{1}{2 \pi} \int_{0}^{2 \pi} \log \|\tilde{f}\|\left(r e^{i \theta}\right) d \theta-N(r, \kappa)+O(1),
$$

where $N(r, \kappa)$ is the averaged counting function of common zeros.

We need a representation whose components have an estimate in terms of $T(r, f)$. Let us start with a reduced representation $f=\left(F_{0}: \ldots: F_{n}\right)$ Consider one-dimensional curves (meromorphic functions) $F_{j} / F_{0}, 1 \leq j \leq n$, We have

$$
T\left(r, F_{j} / F_{0}\right) \leq \frac{1}{2 \pi} \int_{0}^{2 \pi}\left(\log \left|F_{0}\right| \vee \log \left|F_{j}\right|\right)\left(r e^{i \theta}\right) d \theta+O(1)
$$




$$
\leq \frac{1}{2 \pi} \int_{0}^{2 \pi}\left(\log \left|F_{0}\right| \vee \ldots \vee \log \left|F_{n}\right|\right)\left(r e^{i \theta}\right) d \theta+O(1)=T(r, f)+O(1)
$$

By a theorem of J. Miles [14] there exists an absolute constant $C$ and entire functions $G_{j}$ such that

$$
T\left(r, G_{j} F_{j}\right) \leq C T(C r, f), \quad T\left(r, G_{j} F_{0}\right) \leq C T(C r, f), \quad 1 \leq j \leq n .
$$

Thus we obtain a new homogeneous representation $\tilde{f}=\left(f_{0}, \ldots, f_{n}\right)$ where

$$
f_{0}=F_{0}^{n} \prod_{i=1}^{n} G_{i}, \quad f_{j}=F_{0}^{n-1} F_{j} \prod_{i=1}^{n} G_{i}, \quad 1 \leq j \leq n
$$

with the property

$$
T\left(r, f_{j}\right)=O(T(C r, f)), \quad r \rightarrow \infty, \quad 0 \leq j \leq n .
$$

A homogeneous representation with this property will be called good representation. A representation which is simultaneously good and reduced may not exist. From now on we fix some good representation $\tilde{f}$ of our curve $f$.

Given an admissible system $A=\{a\}$, each $a$ being defined by a homogeneous polynomial $P_{a}$ of degree $d_{a}$ we define subharmonic functions

$$
w=\log \|\tilde{f}\|
$$

and

$$
w_{a}=\frac{1}{d_{a}} \log \left|P_{a} \circ \tilde{f}\right|, \quad a \in A .
$$

For every subset $I \subset A,|I|=n+1$ the forms $\left\{P_{a}: a \in A\right\}$ have no common zeros except at the origin (by definition of an admissible system) so for $\|Z\|=1$ we have

$$
C_{1} \leq \bigvee_{a \in I}\left|P_{a}(Z)\right|^{1 / d_{a}} \leq C_{2}
$$

with some constants $C_{1}$ abd $C_{2}$ depending on $I$. By homogeneity we conclude that

$$
\bigvee_{a \in I} w_{a}=w+O(1) \quad \text { for every } I \subset A,|I|=n+1
$$

It follows from (14) that $w$ satisfies the inequality

$$
w(z) \leq C_{1} T\left(C_{2} r, f\right), \quad r=|z|,
$$


with some constants $C_{1}$ and $C_{2}$.

2.2 Pólya peaks and limit functions. Standard references for Pólya peaks are [5] and [2]. Put $T(r)=T(r, f)$ and

$$
\begin{gathered}
\lambda^{*}=\sup \left\{p: \limsup _{t, r \rightarrow \infty} \frac{T(r t)}{r^{p} T(t)}=\infty\right\} ; \\
\lambda_{*}=\inf \left\{p: \liminf _{t, r \rightarrow \infty} \frac{T(r t)}{r^{p} T(t)}=0\right\} .
\end{gathered}
$$

Then $\lambda_{*} \leq \rho \leq \lambda^{*}$ where $\rho$ is defined in (1). So under the assumptions of theorems 1 and 2 we have $\lambda_{*}<\infty$.

For every $\lambda \in\left[\lambda_{*}, \lambda^{*}\right]$ and every $\epsilon_{0} \in(0,1)$ there exists a sequence $r_{k} \rightarrow \infty$ of Pólya peaks of order $\lambda$ that is

$$
T\left(r r_{k}\right) \leq \begin{cases}T\left(r_{k}\right) r^{\lambda+\epsilon_{0}}, & \epsilon_{0}^{-1} \leq r \leq \epsilon_{k}^{-1} \\ T\left(r_{k}\right) r^{\lambda-\epsilon_{0}}, & \epsilon_{k} \leq r \leq \epsilon_{0}\end{cases}
$$

where $\epsilon_{k} \rightarrow 0$.

Now we form the sequences of subharmonic functions

$$
w_{k}(z)=\frac{1}{T\left(r_{k}\right)} w\left(r_{k} z\right)
$$

and

$$
w_{a, k}(z)=\frac{1}{T\left(r_{k}\right)} w_{a}\left(r_{k} z\right), \quad a \in A
$$

These sequences are uniformly bounded from above on compacta in $\mathbf{C}$ in view of (16) and (19). We have $w_{k}(0) \rightarrow 0$ as $k \rightarrow \infty$. By the Convergence Theorem for subharmonic functions (see, for example [11]) we may choose a subsequence of Pólya peaks such that the sequences $\left(w_{k}\right),\left(w_{a, k}\right)$ converge in $L_{\text {loc }}^{1}(d x d y)$ to some subharmonic functions $v$ and $v_{a}, a \in A$. Convergence also holds in $L^{1}$ with respect to one-dimensional measure on every circle (or any smooth curve). The corresponding Riesz measures converge in the topology of $C_{0}^{*}(\mathbf{C})$ that is convergence of measures $\mu_{k} \rightarrow \mu$ means that

$$
\int \phi d \mu_{k} \rightarrow \int \phi d \mu
$$

for every continuous function $\phi$ with compact support. 
The limit subharmonic functions have the following properties:

$$
\begin{gathered}
v(0)=0, \quad v_{a}(0)=0, \quad \text { for } a \in A, \\
v(z) \leq \begin{cases}C|z|^{\lambda+\epsilon_{0}}, \quad|z| \geq \epsilon_{0}^{-1} \\
C|z|^{\lambda-\epsilon_{0}}, & |z| \leq \epsilon_{0}\end{cases}
\end{gathered}
$$

which follow from (16) and (19). Furthermore we conclude from (15) that

$$
\bigvee_{a \in I} v_{a}=v \quad \text { for every } I \subset A,|I|=n+1 \text {. }
$$

2.3 Rescaling of the Riesz measures. For a subharmonic function $u$ we denote by $\mu[u]$ its Riesz measure. For every Borel measure $\mu$ in $\mathbf{C}$ we use the notations

$$
n(r, \mu)=\mu(D(0, r)) \quad \text { and } \quad N(r, \mu)=\int_{0}^{r} n(t, \mu) \frac{d t}{t}
$$

if the last integral is convergent.

The Riesz measures of $w$ and $w_{a}$ have a part in common, namely the measure coming from common zeros of functions in the homogeneous representation. More precisely, denote by $\kappa$ the measure which charges every zero common to all $f_{j}$ according to its multiplicity. Then we have $\kappa \leq \mu[w]$ and $\kappa \leq \mu\left[w_{a}\right], a \in A$. Let $\kappa_{k}$ be defined by

$$
\kappa_{k}(E)=\frac{1}{T\left(r_{k}\right)} \kappa\left(r_{k} E\right)
$$

for every Borel set $E \subset \mathbf{C}$. Then $\kappa_{k} \leq \mu\left[w_{k}\right]$ and $\kappa_{k} \leq \mu\left[w_{a, k}\right], a \in A$. By Jensen's inequality

$$
N\left(r, \kappa_{k}\right) \leq \frac{1}{2 \pi} \int_{0}^{2 \pi} w_{k}\left(r e^{i \theta}\right) d \theta+o(1), \quad r \rightarrow \infty .
$$

Thus $n\left(r, \kappa_{k}\right) \leq N\left(e r, \kappa_{k}\right)$ is bounded for every $r>0$ by a constant independent on $k$. Thus we can select a subsequence of Pólya peaks one more time so that $\kappa_{k} \rightarrow \sigma$, where $\sigma$ is a Borel measure. We have $\sigma \leq \mu[v]$ and $\sigma \leq \mu\left[v_{a}\right]$ for $a \in A$. We also have (see, for example, [8]):

$$
N\left(r, \kappa_{k}\right) \rightarrow N(r, \sigma) .
$$


From (13) we obtain

$$
\Phi(r):=\lim _{k \rightarrow \infty} \frac{T\left(r r_{k}\right)}{T\left(r_{k}\right)}=\frac{1}{2 \pi} \int_{0}^{2 \pi} v\left(r e^{i \theta}\right) d \theta-N(r, \sigma)
$$

and finally from the definition of defect and Jensen's formula

$$
\frac{1}{2 \pi} \int_{0}^{2 \pi}\left(v-v_{a}\right)\left(r e^{i \theta}\right) d \theta=\delta(a, f) \Phi(r) .
$$

3. Proof of Theorem 1 Adding the equalities (24) for all $a \in A$ and using (6) we obtain

$$
\frac{1}{2 \pi} \int_{0}^{2 \pi} \sum_{a \in A}\left(v-v_{a}\right)\left(r e^{i \theta}\right) d \theta=2 n \Phi(r) .
$$

Lemma 1 [9]. Let $v_{1}, \ldots, v_{q}$ and $v$ be subharmonic functions in a region $\Omega, q \geq 2 n$. Assume that

$$
\bigvee_{j \in I} v_{j}=v \quad \text { for every } I \subset\{1, \ldots, q\} \quad \text { with } \quad|I|=n+1
$$

Then

$$
\bigwedge_{I:|I|=n} \sum_{j \in I} v_{j}+n v
$$

is a subharmonic function in $\Omega$.

Our functions $v_{a}$ satisfy the condition (26) in view of (22). We apply this lemma to every finite subset $\left\{v_{a}: a \in J\right\}, J \subset A,|J| \geq 2 n$, and denote by $h_{J}$ the subharmonic function (27). If $A$ is infinite we have a decreasing sequence of subharmonic functions $h_{J}$ which tends to a subharmonic function $h$. This function is not identically equal to $-\infty$ because $h(0)=0$. So

$$
\bigwedge_{|I|=n} \sum_{a \in I} v_{a}+n v=h
$$

From this relation follows that

$$
2 n \sigma \leq \mu[h]
$$


Using (22) we can rewrite (28) as

$$
\sum_{a \in A}\left(v-v_{a}\right)=2 n v-h
$$

We integrate this with respect to $\theta$, then use (25) and (23) to obtain

$$
2 n N(r, \sigma)=\frac{1}{2 \pi} \int_{0}^{2 \pi} h\left(r e^{i \theta}\right) d \theta .
$$

Combined with (29) and Jensen's inequality this implies that $\mu[h]=2 n \sigma$. So the functions

$$
u=v-h /(2 n) \quad \text { and } \quad u_{a}=v_{a}-h /(2 n), \quad a \in A
$$

are subharmonic, they satisfy

$$
\begin{gathered}
\bigvee_{a \in I} u_{a}=u \quad \text { for every } I \subset A,|I|=n+1, \\
\bigwedge_{|I|=n} \sum_{a \in I} u_{a}+n u=0
\end{gathered}
$$

and thus

$$
\sum_{a \in A}\left(u-u_{a}\right)=2 n u
$$

We also have

$$
\Phi(r)=\frac{1}{2 \pi} \int_{0}^{2 \pi} u\left(r e^{i \theta}\right) d \theta
$$

and

$$
u\left(r e^{i \theta}\right) \leq \begin{cases}C r^{\lambda+\epsilon_{0}}, & r \geq \epsilon_{0}^{-1} \\ C r^{\lambda-\epsilon_{0}}, & r \leq \epsilon_{0}\end{cases}
$$

It follows from (33) and (23) that

$$
\frac{1}{2 \pi} \int_{0}^{2 \pi} u\left(e^{i \theta}\right) d \theta=\Phi(1)=1
$$

In particular $u \neq 0$.

Now we derive conclusions from equations (30), (31) and (32). 
1. First notice that each of the two summands in (31) is upper semicontinuous and their sum is continuous. Thus both summands are continuous. In particular $u$ is continuous. The first term in (32) is lower semicontinuous (as a limit of increasing sequence of lower semi-continuous functions) and the second term is continuous. It follows that all summands in the first term are continuous. Thus all $u_{a}$ are continuous.

2. From (32) follows that $u \geq 0$, and (35) implies $u \neq 0$. Let $D$ be a component of the set $\{z: u(z)>0\}$. As $u$ satisfies (34) the number of such components is finite (at most $2 \lambda$ ) by the subharmonic version of the DenjoyCarleman-Ahlfors Theorem [10]. If $u\left(z_{0}\right)=0$ then $u_{a}(z)=0$ for all $a$, this follows from (32). If $u\left(z_{0}\right)>0$ then $u_{a_{0}}\left(z_{0}\right)<0$ for some $a_{0}$. By continuity these inequalities persist in a neighborhood $V$ of the point $z_{0}$. If we define in this neighborhood a new family of subharmonic functions $\left\{u_{a}: a \in A^{\prime}\right\}$, where $A^{\prime}=A \backslash\left\{a_{0}\right\}$ then this new family will satisfy

$$
\bigvee_{a \in I} u_{a}=u \quad \text { for every } I \subset A^{\prime},|I|=n .
$$

Thus Lemma 1 is applicable with $n$ in place of $n+1$ from which we conclude that

$$
h^{\prime}:=\bigwedge_{|I|=n-1, I \subset A^{\prime}} \sum_{a \in I} u_{a}+(n-1) u
$$

is subharmonic. But

$$
u_{a_{0}}+h^{\prime}+u=0 \text { in } V
$$

by (31), that is sum of three subharmonic functions is zero. So we conclude that all these three functions are harmonic.

Thus $u$ is harmonic in every component $D$ of the set $\{z: u(z)>0\}$. By the Maximum Principle for every $a \in A$ there is an alternative: either $u_{a}(z)=u(z), z \in D$ or $u_{a}(z)<u(z), z \in D$. In both cases $u_{a}$ is harmonic in $D$. It follows that if we denote by $I(z)$ the subset of $A$ on which the minimum is attained in (31) at a point $z$, then $I(z)$ is constant in $D$.

Let $\left\{D_{j}\right\}_{j=1}^{q}$ be an enumeration of all components of the set where $u$ is positive. For each $j$ let $I_{j}$ be the constant value of $I(z)$ in $D_{j}$. Define the functions $s_{j}$ in the following way:

$$
s_{j}(z)= \begin{cases}\sum_{a \in I_{j}} u_{a}(z) & \text { if } z \in D_{j} \\ n u(z) & \text { otherwise }\end{cases}
$$


Let us verify that $s_{j}$ are subharmonic in the plane. This needs verification only on the boundary of $D_{j}$. But at every point of this boundary the average property is satisfied trivially, because $u \geq u_{a}$ for all $a$ and all functions are zero on the boundary of $D_{j}$.

Now we define

$$
U_{j}(z)= \begin{cases}n u(z), & z \in D_{j} \\ 0 & \text { otherwise }\end{cases}
$$

Then $U_{j}$ are non-negative subharmonic functions. We have by (31) $s_{j}=$ $\sum_{k=1}^{q} U_{k}-2 U_{j}$. Thus $2 \mu\left[U_{j}\right] \leq \sum_{k=1}^{q} \mu\left[U_{k}\right]$ which implies

$$
2 \bigvee_{j=1}^{q} \mu\left[U_{j}\right] \leq \sum_{k=1}^{q} \mu\left[U_{k}\right] .
$$

Now we are in position to apply the following lemma from [6] (see also [8, Lemma 1], [7]. By support of a function we mean the set where it is different from zero.

Lemma 2 Suppose that non-negative subharmonic functions $U_{1}, \ldots, U_{q}$ have disjoint supports and their Riesz measures satisfy (36). Assume also that (34) is satisfied with $U:=\sum_{j=1}^{q} U_{j}$ in place of $u$ with $\epsilon_{0}<1 / 4$. Then $\lambda$ in (34) is half integer and

$$
U\left(r e^{i \theta}\right)=c r^{\lambda}\left|\cos \lambda\left(\theta-\theta_{0}\right)\right|
$$

for some $c>0$ and $\theta_{0} \in[0,2 \pi)$.

Returning to our function $u=U / n$ and taking into account (35) we obtain from Lemma 2

$$
u\left(r e^{i \theta}\right)=(\pi / 2) r^{\lambda}\left|\cos \lambda\left(\theta-\theta_{0}\right)\right| .
$$

One conclusion is that the set of possible orders $\lambda$ of Pólya peaks is discrete. This implies that $\lambda^{*}=\lambda_{*}=\rho$ in (17) and (18). This means that for every $\epsilon>0$

$$
r^{\rho-\epsilon} T(t) \leq T(r t) \leq r^{\rho+\epsilon} T(t),
$$

as $r$ and $t$ tend to $\infty$ thus every sequence $r_{k} \rightarrow \infty$ can be used as a sequence of Pólya peaks (compare [7]). Thus the above argument is applicable to every sequence, and we conclude that from every sequence one can extract a subsequence such that

$$
\lim _{k \rightarrow \infty} \frac{T\left(r r_{k}\right)}{T\left(r_{k}\right)}=\frac{\pi}{2} r^{\rho},
$$


where $c$ may depend on the sequence. Thus the limit

$$
\lim _{k \rightarrow \infty} \frac{T\left(r r_{k}\right)}{r^{\rho} T\left(r_{k}\right)}
$$

exists uniformly with respect to $r \in[1 / 2,2]$ and the statement (ii) in Theorem 1 follows with $\rho=\lambda$.

Now it follows from (37) that $D_{j}$ are angular sectors and for each $j$ the functions $u_{a}$ are separated into two classes: $n$ of them are equal to $-u$ in $D_{j}$ and the rest are equal to $u$ in $D_{j}$. If we take into account the expression for deficiencies (24) and (23), this proves statement (iii). Finally if one of deficiencies is equal to 1 , the corresponding $u_{a}$ is harmonic, so $\lambda$ has to be an integer because $u_{a}(0)=0$ and $u_{a}(z) \leq u(z) \leq|z|^{\rho}, z \in \mathbf{C}$ and $u_{a} \neq 0$.

4. Proof of Theorem 2. Let us describe the geometric interpretation of functions $w_{a}$ defined in subsection 2.1. We will do this only in the case when our hypersurfaces are non-singular. In this case we can choose the polynomials $P_{a}$ defining our hypersurfaces to be irreducible, and at every point $Z \in \mathbf{C}^{n+1}$ such that $P_{a}(Z)=0$, we have $D P(Z) \neq 0$, where $D=\left(\partial / \partial z_{0}, \ldots, \partial / \partial z_{n}\right)$. It follows that for every such $Z$ there exists a neighborhood $V$ and a positive constant $C$ with the property

$$
C^{-1}<\frac{\operatorname{dist}(W, \tilde{a})}{\left|P_{a}(W)\right|}<C, \quad W \in V
$$

where $\tilde{a}=\pi^{-1}(a)$ is the zero set of $P_{a}$ and dist means the standard distance in $\mathbf{C}^{n+1}$. It follows that for any distance on $\mathbf{P}^{n}$, equivalent to the standard one, we have

$$
C^{-1}<\operatorname{dist}(\pi(Z), a): \frac{\left|P_{a}(Z)\right|}{\|Z\|^{d}}<C, \quad Z \in \mathbf{C}^{n+1}
$$

where $d_{a}=\operatorname{deg} P_{a}$ and the constant $C$ depends only of the chosen distance. Thus the geometric interpretation of the subharmonic functions $w_{a}$ and $w$ introduced in subsection 2.1 is the following:

$$
d_{a}\left(w_{a}(z)-w(z)\right)=\log \operatorname{dist}(f(z), a)+O(1)
$$

where the bound for $O(1)$ depends only of the chosen distance in $\mathbf{P}^{n}$. 
Now we use the notations of the proof of Theorem 1. Fix a sequence of Pólya peaks such that $w_{k} \rightarrow v$ and $w_{a, k} \rightarrow v_{a}$ such that the corresponding functions $u$ and $u_{a}$ satisfy the conclusions made about them in section 3 , in particular, equation (37). Assume for simplicity that $\theta_{0}=0$ in (37) (this always can be achieved by performing a rotation in the plane of independent variable). Put

$$
D=\{z:|\arg z|<\pi /(2 \lambda)\}
$$

Let $a_{1}, \ldots, a_{n} \in A$ be those hypersurfaces for which we have $u_{a}(z)=-u(z), z \in$ $D$. This means that for our sequence of Pólya peaks $r_{k}$ we have

$$
\operatorname{dist}\left(f\left(r_{k} t\right), a_{j}\right) \leq \exp \left\{\left(-\pi d_{a_{j}}+o(1)\right)\left(r_{k} t\right)^{\lambda}\right\}, \quad k \rightarrow \infty, \quad 1 \leq j \leq n
$$

uniformly with respect to $t \in\left[\beta, \beta^{-1}\right]$ for every $0<\beta<1$. In particular, $\operatorname{dist}\left(f\left(r_{k} t\right), a_{j}\right) \rightarrow 0$ for $1 \leq j \leq n$, so after choosing a subsequence of Pólya peaks we can assume that $f\left(r_{k} t\right) \rightarrow Z_{0} \in \cap_{j=1}^{n} a$. Here we used the assumption that our system $A$ is admissible, so the intersection $\cap_{j=1}^{n} a_{j}$ is a finite set.

Now if we assume normal intersection of $a_{1}, \ldots, a_{n}$ at $Z_{0}$ then

$$
\operatorname{dist}\left(W, a_{1}\right) \vee \operatorname{dist}\left(W, a_{2}\right) \vee \ldots \vee \operatorname{dist}\left(W, a_{n}\right)
$$

is equivalent to $\operatorname{dist}\left(W, Z_{0}\right)$ in some neighborhood of $Z_{0}$. So we can conclude from (39) and our assumption that $d_{a} \geq 2$ that

$$
\operatorname{dist}\left(f\left(r_{k} t\right), Z_{0}\right) \leq \exp \left\{(-2 \pi+o(1))\left(r_{k} t\right)^{\lambda}\right\}, \quad k \rightarrow \infty .
$$

To obtain a contradiction consider any hyperplane $H$ containing the point $Z_{0}$ but not containing the whole curve $f(\mathbf{C})$. Let this hyperplane be described by the linear form $P_{H}$. Then for the function $w_{H}=\log \left|P_{H} \circ \tilde{f}\right|$ we consider the limit function $u_{H}$ as it was described in subsections 2.2 and 2.3. We will have $u_{H}(0)=0$ and $u_{H} \leq u$ similarly to the properties of $u_{a}$, and $u$ is given by (37), but on the other hand (40) implies $u_{H}(t) \leq-\pi t^{\lambda}$. This contradicts the Phragmén-Lindelöf theorem (see, for example [12]). So the intersection of $a_{1}, \ldots, a_{n}$ at $Z_{0}$ cannot be normal.

To find another group of hypersurfaces with non-normal intersection we consider adjacent sector

$$
D^{\prime}=\{z:|\arg z-\pi / \lambda|<\pi /(2 \lambda)\}
$$


Let $a_{n+1}, \ldots, a_{2 n}$ be those hypersurfaces in $A$ for which $u_{a}$ are negative in $D^{\prime}$. We can repeat the above argument for $a_{n+1}, \ldots a_{2 n}$ to conclude that their intersection at some point $Z_{1}$ is not normal. It remains to notice that $\left\{a_{1} \ldots, a_{n}\right\} \cap\left\{a_{n+1}, \ldots, a_{2 n}\right\}=\emptyset$ because the subharmonic functions $u_{a}$ cannot be negative in both adjacent sectors.

5. Construction of the Example. Recall that an entire function $y$ is said to have completely regular growth (with respect to order $\lambda$ ) if the limit in $L_{\text {loc }}^{1}$ exists

$$
\lim _{r \rightarrow \infty} r^{-\lambda} \log \left|y\left(r t e^{i \theta}\right)\right|=t^{\lambda} h(\theta) .
$$

The function $h$ is called indicator of $y$ (see, for example [12]).

Consider the differential equation

$$
y^{\prime \prime \prime}-z y^{\prime}-y=0 .
$$

It is equivalent to the family of non-homogeneous Airy equations

$$
y^{\prime \prime}-z y=c, \quad c \in \mathbf{C} .
$$

When $c=0$ there are three solutions, of (42), called Airy functions [1, sect 10.4] $y_{0}, y_{1}$ and $y_{2}$, with the property $y_{0}+y_{1}+y_{2}=0$ and such that any two of them are linearly independent. From the well-known asymptotic expansions of Airy functions (see, for example equation (10.4.59) in [1]) one can find the indicators ${ }^{2}$ of all solutions of (41), using the method of variation of constants. Possible indicators of solutions of (41) are the following.

First of all we have a two-dimensional space of solutions of (42) with $c=0$. Their indicators are

$$
H_{0}(\theta)=-\cos \left(\frac{3}{2} \theta\right), \quad|\theta| \leq \pi ; \quad \text { and } \quad H_{j}(\theta)=H_{0}(\theta \pm \pi / 3), \quad j=1,2 .
$$

The rest of solutions of (41) which correspond to non-zero values of $c$ in (42) may have indicators from the following list:

$$
G_{0}(\theta)=\left(-\cos \left(\frac{3}{2} \theta\right)\right)^{+}, \quad|\theta| \leq \pi ; \quad \text { and } \quad G_{j}(\theta)=G_{0}(\theta \pm \pi / 3), \quad j=1,2 .
$$

\footnotetext{
${ }^{2}$ All solutions of differential equations (11) with polynomial coefficients are entire functions of completely regular growth [16]. For the equation (41) this can be seen directly from the asymptotic expansions of Airy functions.
} 
Notice that for every $\theta \in[-\pi, \pi]$ the set of solutions whose indicator is strictly negative at $\theta$ is at most one-dimensional, and the set of solutions whose indicator is non-positive at $\theta$ is at most two-dimensional.

Now let $f$ be the holomorphic curve whose homogeneous coordinates are any three linearly independent solutions of (41). If $A=\left\{a_{1}, \ldots, a_{q}\right\}$ is an admissible system of hyperplanes, and $P_{j}$ are the corresponding linear forms then $g_{j}=P_{j} \circ \tilde{f}, 1 \leq j \leq q$ is a system of solutions of (41) having the property that every three of these solutions are linearly independent. Let $h_{j}$ be the indicators of these solutions $g_{j}$. and $h$ be their pointwise maximum,

$$
h(\theta)=|\cos ((3 / 2) \theta)|, \quad|\theta| \leq \pi .
$$

Then we have

$$
\int_{-\pi}^{\pi} h=3 \int_{-\pi / 3}^{\pi / 3} \cos \left(\frac{3}{2} \theta\right) d \theta=4
$$

We claim that

$$
\sum_{j=1}^{q} \int_{-\pi}^{\pi}\left(h-h_{j}\right) \leq 8 \int_{-\pi / 3}^{\pi / 3} \cos \left(\frac{3}{2} \theta\right) d \theta=\frac{32}{3} .
$$

This follows from the fact that in each of the three components of the set $\{\theta: h(\theta)>0\}$ at most one indicator can be negative and at most two can be non-positive, but in addition we cannot have negative indicators in all three components because solutions $y_{0}, y_{1}$ and $y_{2}$ with indicators (43) are linearly dependent.

So we have

$$
\sum_{j=1}^{q} \int_{-\pi}^{\pi}\left(h-h_{j}\right) \leq \frac{8}{3} \int_{-\pi}^{\pi} h
$$

which implies that

$$
\sum_{j=1}^{q} \delta\left(a_{j}, f\right) \leq \frac{8}{3}
$$

\section{References}

[1] M. Abramowitz and I. Stegun, Handbook of Mathematical Functions, Dover, NY, 1965. 
[2] N. H. Bingham, C. M. Goldie and J. L. Teugels. Regular variation. Encyclopedia of Mathematics and its Applications, 27. Cambridge University Press, Cambridge-New York, 1989.

[3] H. Cartan, Sur les zéros des combinations linéaires de $p$ fonctions holomorphes données, Mathematica (Cluj), 7 (1933), 5-31.

[4] D. Drasin, Proof of a conjecture of F. Nevanlinna concerning functions which have deficiency sum two, Acta Math., 1987, 158, 1-94.

[5] D. Drasin and D. Shea, Pólya peaks and oscillation of positive functions, Proc. Amer. math. Soc., 34 (1972), 403-411.

[6] A. Eremenko, New proof of Drasin's theorem on meromorphic functions of finite order with maximal deficiency sum I, II, Function Theory, Functional Analysis and their Applications, (1989), 51, 107-116 and 52, 69-77. English translation: Journal of Soviet Math., 52, 6 (1990), 3522-3529 and 52, 5 (1990), 3397-3403.

[7] A. Eremenko, Meromorphic functions with small ramification, Indiana Univ. math, J., 42, 4 (1993), 1193-1218.

[8] A. Eremenko and M. Sodin, On meromorphic functions of finite order with maximal deficiency sum. Function Theory, Functional Analysis and their Applications. 55, (1991) 84-95 (English translation: J. Soviet Math., 59 (1992), 643-651.)

[9] A. Eremenko and M. Sodin, Value distribution of meromorphic functions and meromorphic curves from the point of view of potential theory. St. Petersburg Math. J., 3 (1992), 109-136.

[10] W. Hayman and P. Kennedy, Subharmonic Functions, vol. 1 Academic Press, NY, 1976.

[11] L. Hörmander, The analysis of linear partial differential operators. I. Distribution theory and Fourier analysis. Second edition. Springer-Verlag, Berlin, 1990.

[12] B. Ya. Levin, Lectures on entire functions, Translations of Math. Monographs, vol. 150, AMS, Providence, RI, 1996. 
[13] S. Lang, Introduction to Complex Hyperbolic Geometry, Springer, NY, 1987.

[14] J. Miles, Quotient representations of meromorphic functions. J. Analyse Math. 25 (1972), 371-388.

[15] E. I. Nochka, On the theory of meromorphic curves, Soviet Math. Dokl. 27 (1983), no. 2, 377-381.

[16] V. P. Petrenko, Entire Curves (Russian) "Vishcha Shkola", Kharkov, 1984.

[17] N. Toda, On the order of holomorphic curves with maximal deficiency sum, Kodai Math. J. 18 (1995), no. 3, 451-474.

Purdue University,West Lafayette IN 47907; eremenko@math.purdue.edu 